\title{
Target-distractor discriminability in visual search
}

\author{
HAROLD PASHLER \\ University of California, San Diego, La Jolla, Califormia
}

\begin{abstract}
Previous research has demonstrated that discriminability of target and background elements has powerful effects on visual search performance. Typically, discriminability has been manipulated between blocks or subjects, allowing subjects to anticipate the discriminability in advance of each trial, and the results, therefore, have been consistent with a wide range of models. Two experiments are reported in which the number of distractors similar to the target was varied from trial to trial, preventing such anticipation. In Experiment 1, subjects' response times increased as target-confusable distractors were added, for both "yes" and "no" responses. In Experiment 2, subjects searched for either of two targets. Distractors similar to one target slowed down detection of either that target or the other target, to similar degrees. The results indicate that decision noise, rather than feature-specific inhibition, is the major source of the latency effects. The results do not support models proposing that decisions in speeded search are based upon information integrated across all positions in the display. The data also would require (perhaps implausible) modification of the independent-channels model. They are broadly consistent, however, with models hypothesizing a parallel search followed by some slow serial checking, such as that developed by Hoffman (1978).
\end{abstract}

In visual search tasks, subjects attempt to detect the presence of prespecified target(s) in arrays of visually presented elements. The task has been intensively studied for several reasons. Among them is that it provides a close, but experimentally controlled, analogue of an extraordinarily common and important behavior: detecting and orienting to visual stimuli of particular interest. Another is that it permits the experimenter to examine the time course of visual recognition without requiring that the subject retain or report more than one element; such requirements seem to introduce special limitations of their own (Duncan, 1980b; Estes \& Taylor, 1964). Previous research has found target-background similarity to have major effects on visual search performance; the purpose of the present article is to point out and rectify a major gap in the empirical characterization of the similarity effects, and to explore the implications for the architecture of visual processing.

Research on visual search has focused heavily on the effects of the number of items in a display (display size) on reaction times (RTs) and on accuracy to detect target(s) or to indicate the absence of a target. Increases in display size produce a robust decrement in accuracy and speed of response (Estes \& Taylor, 1964). By itself, this effect is consistent with a wide range of possible task organizations (Duncan, 1980a). It is necessary, therefore, to employ additional manipulations in order to better understand

The author is grateful to Peter Badgio, Jonathan Baron, Dorrit Billman, James C. Johnston, and Jeff Miller for useful comments and discussions. Correspondence should be addressed to the author at the Department of Psychology, C-009, University of California, San Diego, La Jolla, CA 92093. the processing that underlies search, and a wide range of approaches have been employed.

Several researchers have observed that the similarity of target and background items has a major effect on performance in visual search. For instance, Estes (1972) had subjects perform a forced-choice letter-detection task in which the background items consisted of either disks (dissimilar condition) or letters (similar condition). Accuracy was poorer and latencies longer with the similar background items. Corcoran and Jackson (1977) observed a similar pattern of results, comparing different background elements in speeded detection tasks. More recently, Duncan (1983) and Krueger (1984) presented data suggesting that performance differences between within-category search (e.g., a letter target among letters) and betweencategory search (e.g., a digit among letters) may well be accounted for by differences in relative target-background discriminability (in the case of single-target search only).

In each of these experiments, target-background similarity was held constant within blocks or trials, or even manipulated between subjects. As a result, subjects could anticipate in advance of each trial whether or not distractors would consist of target-confusable items. It seems that manipulation of discriminability between trials, so as to prevent anticipation of the discriminability level on a given trial, may yield information that may be diagnostic concerning a variety of major hypotheses about visual search.

A number of different accounts of the target-distractor similarity effect have been suggested. Estes (1972) proposed that the impaired performance in the similardistractor condition resulted from competition for a limited number of feature detectors for particular features within any given spatial region (called the interactive-channels 
hypothesis). The scarcity of feature detectors would effectively generate inhibition in the recognition of spatially adjacent forms that are visually similar.

An alternative framework was suggested by Eriksen and Spencer (1969), and later developed by Gardner (1972) and Shiffrin and Geisler (1973). These authors pointed out that a deterioration in accuracy of performance with increases in target-background similarity would, on certain assumptions, arise for purely statistical reasons. Even if each element in a display is handled independently and without attentional limitations, increases in confusability may degrade performance by increasing the chances of a false alarm. Essentially, this view proposes that the effects of target-distractor discriminability originate in decision noise; unlike Estes's (1972) hypothesis, this account need not invoke effects within the character recognition process itself.

The decision noise account of discriminability effects in latencies may be consistent with either serial or parallel perceptual processing of the items in the display. The serial case is straightforward: if more time were spent determining whether confusable items in the array are or are not targets, then RTs for both "yes" and "no" responses should be increased linearly with the number of confusable distractors. If all the items are handled in parallel, the situation is slightly more complex. One model that has received some support suggests a parallel decision process based on a sum of the evidence favoring target presence, integrated from all display positions (Kinchla, 1974); henceforth, I call this the integration model. An alternative is the independent-channels model (Shiffrin \& Geisler, 1973). According to this model, each channel is analyzed separately, and a detection response can occur when a channel reaches threshold. Either of these parallel models can account for discriminability effects when the subject can anticipate the discriminability of the distractors on a given trial, because subjects can set their criteria higher when difficult discriminations will be required.

It can be seen, then, that effects of discriminability, when this variable is blocked, are consistent with a host of possible accounts. In Experiment 1, we look for a discriminability effect when the number of target-similar distractors is changed from trial to trial in a manner that is unpredictable to the subject. Then, in Experiment 2, we ask whether discriminability effects on positive (targetpresent) trials stem from the similarity of the distractors to the target actually present, or from the similarity of the distractors to the complete set of potential targets. The General Discussion explores the constraints posed by the data on the class of possible visual search models.

\section{EXPERIMENT 1}

In Experiment 1, subjects searched for a target letter and pressed one button if it was present, another if it was not. Circular displays consisted of six items, with the number of target-confusable and target-nonconfusable distrac- tors in each display varying between trials. Thus the primary factors of interest are target presence/absence and number of target-confusable distractors $(0,1,3$, or 5$)$.

\section{Method}

Subjects. Nine college students were paid to serve as subjects in two 1-h sessions.

Design. Each session consisted of 1 practice block plus $10 \mathrm{ex}$ perimental blocks, each composed of 64 trials. In each block, there were 8 trials in each of the eight conditions, corresponding to two levels of target presence/absence $\times$ four levels of target-similar distractors $(0,1,3$, and 5 items). The trials in a block appeared in random order.

Apparatus. Stimuli were presented on an Amdek Color-I Monitor, controlled by microcomputer (allowing millisecond response timing and synchrony with the display refresh cycle). Responses were made by depressing one of two microswitch buttons on a panel resting on the table in front of the subject. Subjects sat approximately $60 \mathrm{~cm}$ from the screen and used their left and right index fingers for the target-absent and target-present responses, respectively.

Stimuhi. All stimuli were capital letters. For all subjects, the target was the letter $C$. The similar distractors were Gs, and the dissimilar distractors were Xs and Ls. The selection of the stimuli was based upon the interconfusability matrix for capital letters reported by van der Heijden, Malhas, and van den Roovart (1984), who presented a carefully collected empirical interletter confusion matrix for continuous-line capital letters. For this they used a stimulus set that appears very similar to that employed in the present study (the most notable difference appears to be the shortened middle horizontal segment on the $E$ in our character set). The probabilities of C, G, $X$, and $L$ being reported as $C$ in the data of van der Heijden et al. were $.712, .050, .000$, and .013 , respectively; thus a substantial confusability difference is provided.

The arrays were composed as follows. If the display included a target, it was placed in a randomly chosen position. Then Gs were placed in randomly chosen remaining positions, according to how many confusable distractors were required. The remaining positions were filled with Xs and Ls, chosen randomly with replacement.

Each display consisted of six characters, presented in a circle approximately $3.7 \mathrm{~cm}$ in outer diameter. Based on a typical viewing distance of $60 \mathrm{~cm}$, this corresponded to about $3.5^{\circ}$ visual angle. The characters were presented on the CRT in high-intensity white, against a black background. Each character was composed out of a square of $7 \times 7$ dots, and measured approximately $.7 \times .4 \mathrm{~cm}$ vertically and horizontally (about $.7^{\circ} \times .4^{\circ}$ visual angle).

Procedure. Each trial began with the presentation of a central fixation point (a plus sign). The fixation point remained in view for $.5 \mathrm{sec}$, followed by $.5 \mathrm{sec}$ of blank screen. The display of six characters then appeared, and remained on the screen until the subject made a response. The interval between the response and the fixation point for the following trial was approximately $1.5 \mathrm{sec}$.

The subjects were instructed that they should respond as quickly and accurately as possible, and that they should not make more than "a few" errors on any given block. The practice block was included in both sessions, but not scored. A rest period separated the blocks, and cumulative feedback was presented at that time, consisting of mean correct RT and number of errors for the current and preceding blocks. Subjects were instructed to rest as long as they wished, and to initiate the next block by pressing the space bar on the microcomputer.

\section{Results}

Any RTs greater than 1,200 msec or less than $150 \mathrm{msec}$ were discarded (but not treated as errors). The mean cor- 




Figure 1. Mean reaction times in Experiment 1. Dotted lines: target absent. Solid lines: target present.

rect RTs are shown in Figure 1, and the error rates are reported in Table 1. The results for both sessions show plainly that the increase in the number of target-confusable distractors slowed response times, and more so for the "no" responses than for the "yes" responses. The effect of target presence/absence was significant $[F(1,8)=$ $49.3, p<.0001]$, as were the effects of number of confusable distractors $[F(3,24)=48.9, p<.0001]$ and sessions $[F(1,8)=51.6, p<.0001]$. The interaction of number of confusable distractors with target presence/ absence was significant $[F(3,24)=21.3, p<.0001]$, reflecting the larger effect of distractors on target-absent responses. Sessions interacted with number of confusable distractors $[F(3,24)=9.1, p<.0005]$, reflecting a smaller distractor effect on the second session. No other interactions were significant.

The error rates were analyzed in the same way. The effect of target presence/absence was significant $[F(1,8)$

Table 1

Percent Error Rates in Experiment 1

\begin{tabular}{lrrrr}
\hline & \multicolumn{4}{c}{ Number of Confusable Distractors } \\
\cline { 2 - 5 } & 0 & 1 & 3 & 5 \\
\hline & \multicolumn{5}{c}{ Day 1 } & & \\
Target Present & 4.3 & 4.3 & 5.4 & 2.9 \\
Target Absent & 0.6 & 3.1 & 3.6 & 2.5 \\
& \multicolumn{5}{c}{ Day 2 } & & \\
Target Present & 3.7 & 5.5 & 5.3 & 3.7 \\
Target Absent & 0.8 & 2.2 & 4.0 & 4.0 \\
\hline
\end{tabular}

$=29.8, p<.0001]$, as was the effect of number of confusable distractors $[F(3,24)=5.1, p<.01]$. The other effects were nonsignificant.

\section{Discussion}

The results indicate that target-confusable distractors increase latencies to detect targets, even when the number of such distractors is not known in advance by the subject. Evidently, the effect on latencies is not simply the result of subjects' strategies of setting a higher criterion for detection prior to each trail, on the basis of an expectation of these distractors. The effects of similar distractors are present in both target-present and target-absent responses, although they are larger when no target is present. The effects on error rates are modest, taking the form of an increase in both misses and false alarms.

\section{EXPERIMENT 2}

Experiment 2 was designed to investigate further the effect of target-similar distractors on target detection. In this experiment, subjects searched for two targets, pressing one button when either of the targets was present, and the other button when neither was present (only one target could be present on a given trial). Two target-similar distractors were employed, one similar to each of the possible targets. The major question of interest here concerns the effect of distractors confusable with one target on latency and on accuracy to detect the other target. 


\section{Method}

Subjects. Eighteen college students served as subjects in return for payment. Twelve subjects served in two $1-h$ sessions for the main experiment, and 6 additional subjects participated in a single session for the control condition.

Design. Each session consisted of 1 practice block plus 10 experimental blocks, each composed of 60 trials. Half the trials in each block contained a target, and half did not. Each of the two targets appeared on half the target-present trials, and within the set of trials containing a given target, five conditions appeared equally often: no similar distractors, 2 or 4 instances of one similar distractor, and 2 or 4 instances of the other similar distractor. The target-absent trials were similarly divided into five conditions depending upon distractor composition. Accordingly, in each block there were three trials per target-present condition and six trials per target-absent condition.

Apparatus. The same apparatus was used in this experiment as in the previous one.

Stimuli. All stimuli were capital letters. For all subjects, the targets were the letters $\mathbf{C}$ and $\mathbf{E}$. The similar distractors were $G$ s and Fs, and the dissimilar distractors were Xs and Ns. The selection of the stimuli was based upon the interconfusability matrix for capital letters reported by van der Heijden et al. (1984). In their data, the probabilities of $\mathbf{C}, \mathrm{E}, \mathrm{G}, \mathrm{F}, \mathrm{X}$, and $\mathrm{N}$ being reported as $\mathrm{C}$ were $.712, .022, .050, .011, .000$, and .001 , respectively. The probabilities of each of these characters being reported as $E$ were .049 , $.350, .018, .057, .002$, and .001 , respectively. The selection of letters was intended to accomplish several objectives simultaneously, insofar as possible. Each confusable distractor-target pair (G-C, F-E) has roughly comparable internal confusion rates (.050, .057), and the crosswise distractor-target confusion rates are lower and fairly comparable $(.011, .018)$. The nonconfusable distractors have quite comparable and very low confusability with the two targets. There is a notable difference in report accuracy for $C$ and $E$ in van der Heijden et al.'s data set. This might reflect "intrinsic" difficulties in identifying the $\mathrm{E}$ compared with the $\mathrm{C}$, as well as higher average confusability of the rest of the alphabet with $E$. However, the selection seemed optimal given the limited number of letters to choose among. The displays were composed as in Experiment 1.

Procedure. The procedure followed that of Experiment 1.

\section{Results}

Any RTs greater than 1,200 msec or less than $150 \mathrm{msec}$ were discarded (but not treated as errors). The mean correct RTs are shown in Figure 2, and the error rates are reported in Table 2. The results for both sessions show plainly that the increase in the number of target-confusable distractors on a trial-to-trial basis slowed response times, and more so for the "no" responses than for the "yes" responses. This replicates the results of Experiment 1.

In order to compare the effects of each of the two different distractors on detection of the two targets, the "yes" responses were analyzed as follows. The slopes of the functions relating detection latencies to number of targetsimilar distractors were computed by least-squares estimate. Note that these slopes correspond to the eight lines contained on the bottom four panels of Figure 2. On Day 1 , the increases for detecting a $C$ averaged 20.7 and $37.9 \mathrm{msec} /$ distractor for distractors $F$ and $G$, respectively; for detecting an $E$, the increases averaged 17.2 and 29.3

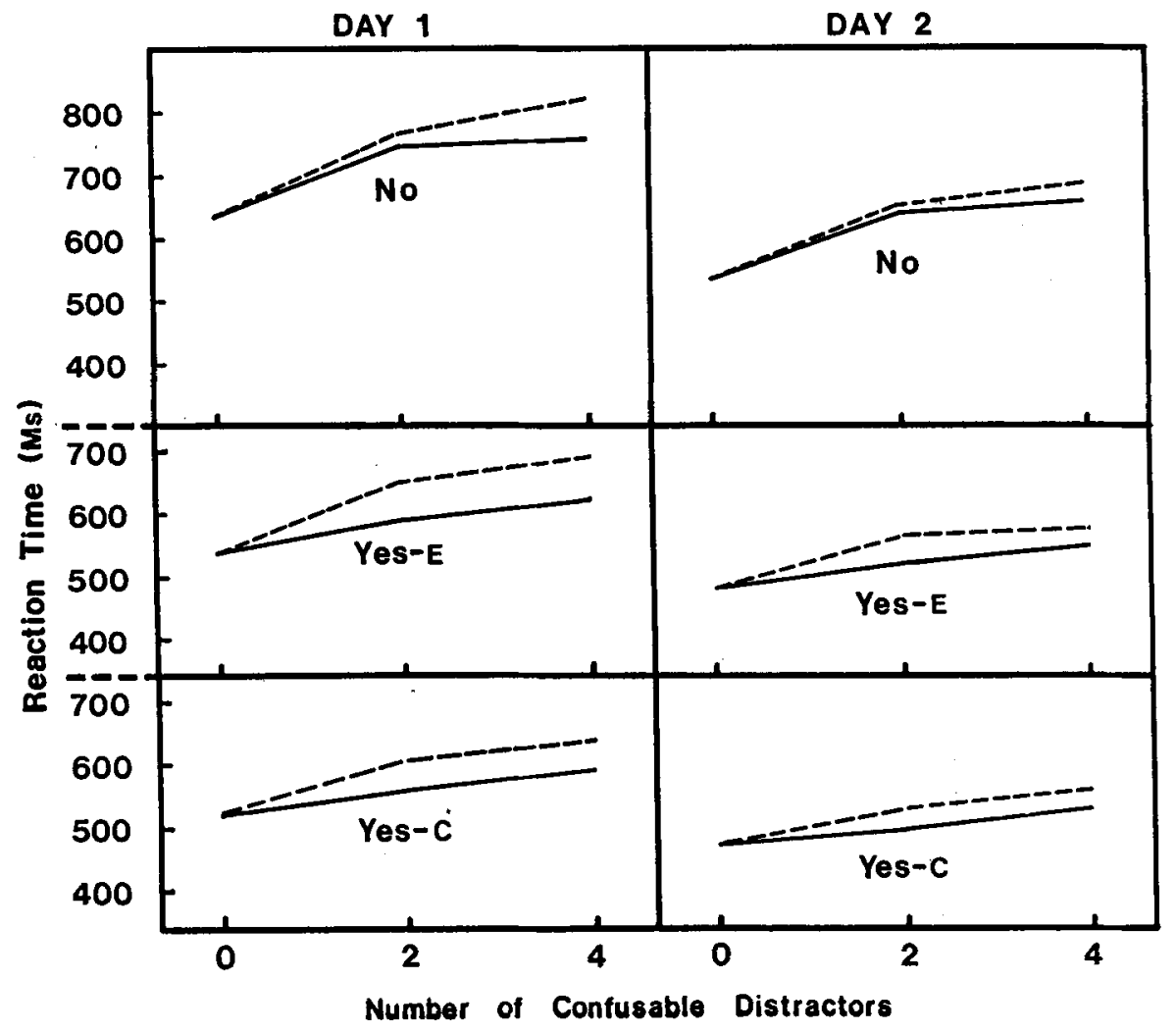

Figure 2. Mean reaction times in Experiment 2. Top panels: Target absent. Middle panels: Target $\mathbf{E}$ present. Bottom panels: Target $\mathrm{C}$ present. Dotted lines: Parameter is number of Gs in display. Solid lines: Parameter is number of Fs in display. 
msec/distractor for distractors $F$ and $G$, respectively. On Day 2, the corresponding increases were 17.2, 24.1, 14.0, and $21.1 \mathrm{msec} /$ distractor.

An analysis of variance was conducted on the slopes. The effect of identity of the target-confusable distractor (F vs. G) was significant $[F(1,11)=5.95, p<.01]$, reflecting greater effects of adding Gs than of adding Fs. The effect of target detected (E vs. C) was also significant $[F(1,11)=10.9, p<.01]$, reflecting steeper slopes in detecting Es than in detecting Cs. Most importantly, the target and distractor effects did not interact $(F<1)$. The effect of session was significant $[F(1,11)=11.8$, $p<.01]$. It interacted significantly with distractor $[F(1,11)=5.3, p<.05]$ but not with target. Other interactions were also nonsignificant.

\section{Discussion}

The results from this experiment are quite clear. A between-trials manipulation of the number of distractors similar to one target caused a similar delay in target detection for detection of both that target and the other target in the set. As in the previous experiment, the effect of target-similar distractors is larger in the target-absent latencies than in the target-present latencies.

The results seem to indicate that adding distractors similar to one target delayed detection of the other target, an effect comparable to the effect of a distractor similar to the detected target. Before accepting this conclusion, one might reasonably want assurance that it is indeed the similarity, for example, of distractor $G$ to target $C$ that delays detection of target $E$ when Gs are added to the display. For this purpose, a control condition was run with 6 subjects. The procedure was identical to that described above, with a few exceptions. The subjects searched for only a single target: E. The trials containing the other target-C-were omitted from the experiment, so twice as much data per positive condition was collected on each session.

Accordingly, it is possible to compare the effect of adding the two distractors $\mathrm{F}$ and $\mathrm{G}$ on detection of the target $E$ when subjects either (1) are looking only for an $E$ (control) or (2) are looking for either an $\mathrm{E}$ or a $\mathrm{C}$ (main experiment). The mean reaction times and error rates for the control condition are shown in Table 3. The latencies indicate that when subjects looked for an $E$ only, the

Table 2

Percent Error Rates in Experiment 2

\begin{tabular}{|c|c|c|c|c|c|}
\hline & \multicolumn{5}{|c|}{ Number of Confusable Distractors } \\
\hline & $\mathbf{0}$ & $2 \mathrm{Fs}$ & $4 \mathrm{Fs}$ & $2 \mathrm{Gs}$ & $4 \mathrm{Gs}$ \\
\hline \multicolumn{6}{|c|}{ Day 1} \\
\hline E Present & 2.6 & 1.9 & 3.6 & 4.7 & 11.7 \\
\hline C Present & 2.1 & 4.0 & 2.9 & 6.5 & 10.8 \\
\hline No Target & 0.6 & 1.7 & 1.1 & 3.5 & 1.4 \\
\hline \multicolumn{6}{|c|}{ Day 2} \\
\hline E Present & 2.5 & 2.2 & 4.2 & 5.3 & 7.5 \\
\hline C Present & 1.7 & 5.6 & 2.5 & 1.7 & 7.2 \\
\hline No Target & 0.6 & 1.7 & 1.9 & 1.9 & 1.9 \\
\hline
\end{tabular}

Table 3

Mean Reaction Times and Percent Error Rates in Control Condition

Number of Confusable Distractors

\begin{tabular}{lllll}
\hline 0 & $2 \mathrm{Fs}$ & $4 \mathrm{Fs}$ & $2 \mathrm{Gs}$ & $4 \mathrm{Gs}$ \\
\hline
\end{tabular}
Reaction Times

\begin{tabular}{llllll} 
E Present & 490 & 548 & 586 & 539 & 533 \\
No Target & 587 & 699 & 717 & 613 & 613 \\
& \multicolumn{5}{c}{ Error Rates } \\
E Present & 2.7 & 6.7 & 7.8 & 7.2 & 8.9 \\
No Target & 1.7 & 7.2 & 3.9 & 4.4 & 1.1 \\
\hline
\end{tabular}

presence of Fs had a much more deleterious effect than did the presence of Gs, the reverse of the effect observed when subjects looked for either $\mathrm{E}$ or $\mathrm{C}$ (main experiment). Interestingly, the Gs still did have an effect, which should not be surprising given the confusabilities reported above, that is, that $\mathrm{G}$ is more confusable with $\mathrm{E}$ than is $\mathrm{X}$ or $\mathrm{N}$.

To assess the reliability of the interaction between instructional condition and identity of confusable distractor (i.e., the contrast between the results in the main experiment and those in the control condition), the least-squares slopes relating RTs to number of confusable distractors were estimated for each subject, as described in the Results section above. On the "yes" responses, the effects of adding Fs and adding Gs averaged 23.9 and $10.5 \mathrm{msec} /$ item, respectively. On the "no" responses, the effects averaged 32.5 and $6.6 \mathrm{msec} / \mathrm{item}$, respectively. The differences between the slope for Fs and the slope for Gs were calculated separately for each subject; they ranged between +7.0 and +17.0 for "yes" responses, and +11.7 and +40.2 for "no" responses. The same slope differences were calculated on the data from all 12 experimental subjects' main experimental sessions. The differences (F-slope minus G-slope) ranged between +3.5 and -45.0 for the "yes" responses and +10.1 and -38.4 for the "no" responses. For both "yes" and "no" responses, these difference distributions are entirely nonoverlapping, and thus each is significant by planned Mann-Whitney $U$ comparison, at $p<.0001$.

\section{GENERAL DISCUSSION}

The present results bear directly on a number of questions about the mechanisms and strategies underlying visual search performance. Several basic results were reported. First, when the number of distractors visually similar to a target was manipulated between trials, to prevent subjects from anticipating the presence of confusable distractors, a highly reliable increase in RTs resulted. Second, the effect was significantly larger on negative trials than on positive trials. Finally, in Experiment 2, subjects searched for either of two targets; when a given target was present, there might be distractors similar to it or distractors similar to the other target (that was not present). Both types of confusable distractors produced very similar effects; that is, distractors similar to an (ab- 
sent) target slowed detection of an alternative target that was present.

Several conclusions can be drawn. The interference effect of target-similar distractors on detection performance (in speeded tasks with ample stimulus exposures) cannot to any major degree be due to perceptual interactions of the sort proposed by Estes (1972). Estes suggested that similar items produce mutual inhibition, due to competition for a limited number of feature detectors tuned to any particular feature. This suggestion was made largely on the basis of the between-blocks target-distractor confusability effect on latencies reported by Estes (1972). If perceptual interference were responsible for the effect, then it should be abolished in the condition in Experiment 2 in which target similarity to the other target was manipulated. However, the effect was intact in these conditions.

The present conclusion agrees with other recent work that calls into question the role of feature-specific interference, at least where display density is moderate or low. Gilmore (1980) found no effect of distractor similarity in a paradigm requiring subjects to name an item presented in a prespecified location, flanked by distractors slightly over $1^{\circ}$ away. Santee and Egeth (1982) found interference in classifying a character when a duplicate character was adjacent to it, but this interference appeared only in report accuracy in a data-limited situation. In contrast, latency of speeded response with adequate stimulus exposure was facilitated (suggesting interactions at the level of response selection), and the authors suggested that feature-specific interference, if it exists, may be restricted to the data-limited situation. However, it appears that even the identical flanker effect in data-limited conditions may not actually reflect featural interference, since Egeth and Santee (1981) observed that the effect persists when the interfering neighbor has the same letter identity, but is a visually dissimilar case switch (e.g., $A$ and a). Finally, Estes (1982) has reported evidence suggesting that similarity effects in report tasks are largely a function of various biases that are generated by the flanking elements. In summary, the work cited suggests that perceptual or conceptual similarity of neighboring elements can have damaging effects in report tasks, but the effects are not especially robust, and may arise as a strategic reaction to location uncertainty or other factors peculiar to the report situation. The results reported here argue that the robust response slowing caused by target-similar distractors in speeded search tasks - the phenomenon that originally motivated the postulation of feature-specific interference-is not caused, to any substantial degree, by that sort of perceptual effect.

As an account of the distractor similarity effect, the natural alternative to interference in the perceptual processing is interference-specifically, noise-in the decision process (Gardner, 1972). The general principle that similar items are distinguished more slowly applies across a wide range of tasks and materials. Indeed, some decrement in the accuracy of performance is, on certain assumptions, statistically inevitable. However, what is of interest here is how subjects adjust to such a manipulation when they cannot anticipate its presence. The effects reported contradict a number of plausible accounts of how evidence might be accumulated for selecting a response. In general, they seem to suggest more sophisticated strategic control than has been previously suggested (except in the model of Hoffman, 1978, discussed below).

As described above, Kinchla (1974) proposed a model according to which information consistent with target presence accrues on each channel, and this information is summed in a central representation that forms the basis for the decision process. Presuming that the distributions of evidence on each channel, conditioned on target presence or absence, have nonzero variability, then several predictions follow for statistical reasons. The accuracy that is possible (i.e., even for an optimal decision process) will be reduced with increases in display size and with increases in target-background confusability. This accords with the results of numerous studies, and Kinchla obtained a satisfactory quantitative fit to such a data set.

The extension of the model to speeded performance is straightforward. Positive responses may be selected if the summed target-evidence variable exceeds a preset threshold. On target-present trials, the presence of distractors similar to the target should therefore speed the positive response, since they should increase the evidence for target presence, summed over display positions. Thus this model clearly makes the wrong predictions in both of the current experiments, because similar distractors increase, rather than decrease, positive RTs. Furthermore, it is not clear that there is any way to modify the model to fit the data, given the key assumption that the decision process has access only to pooled information.

An alternative parallel model suggests that evidence accrues independently and in parallel on each channel, with a detection response based upon the first detection occurring on any of the channels (e.g., Eriksen \& Spencer, 1969; Shiffrin \& Geisler, 1973). If the detection criteria are preset before each trial, then similar distractors should not slow down correct positive responses. They could have several other effects, however. They could increase false alarm errors on negative trials, or increase latencies on negative trials (if it took longer to reject similar distractors). On positive trials, the similar distractors could even speed up responses, if they were mistaken for targets often enough and quickly enough to provide statistical facilitation (by "beating" the real target in the race process). Given the robust slowing of correct positive RTs due to similar distractors, the data presented above clearly undermine this conception of the task.

There are various purely serial models that might be suggested. For instance, a serial scan of the array, in which each item in tum is compared with the target, might be operating. The determination might take longer for elements similar to the target, thus increasing latencies. In its simplest form, such a model predicts that latencies should be a linear function of the number of target-similar distractors in the display, with the slope for "no" trials 
twice that for "yes" trials, presuming self-termination. In the present data, the functions depart markedly from linearity, exhibiting distinct negative acceleration; this does not support such a model. But more generally, a variety of other experiments provide a strong basis for doubting that such rapid serial scanning of the display occurs in target-detection situations (Duncan, 1980b; Pashler \& Badgio, 1985; Shiffrin \& Gardner, 1972).

What could explain this data, then? Several alternatives seem to be viable. The first one is a modification of the independent-channels model (Gardner, 1972). Rather than presuming that the criterion for target detection on each channel is preset in advance of the trial, one might suggest that it is dynamically adjusted as evidence begins to accrue. The subject may take notice of the fact that on many trials, several distractors may be present that are easily confused with the target. Therefore, a strategy might be adopted of raising the detection threshold on all channels if evidence for target presence is accruing on several channels. This account cannot be definitively rejected, but several considerations may weaken its plausibility. First, it supposes extremely rapid strategic adjustments of a task-specific sort. Research on speeded implementation of simple stimulus-dependent strategies would not lead one to expect this sort of rapid flexible change in strategies (Logan \& Zbrodoff, 1982; Logan, Zbrodoff, \& Fostey, 1983). Second, the results of Experiment 2 argue that if such adjustment occurs, it alters the criteria for detecting either target in disjunctive search, which would plainly be nonoptimal. Nonetheless, this model cannot be ruled out.

An alternative approach would hypothesize some kind of "checking" process, following extensive parallel processing. The checking process may be employed in order to deal with the problem of decision noise. The effect of target-similar distractors may arise in such a checking stage. Hoffman $(1978,1979)$ proposed a model of visual search that incorporates precisely this idea, and that can be said to predict the basic results reported in this article. Hoffman suggested that the initial processing takes the form of a parallel analysis of the display, which determines for each display position some estimate of its similarity to the target. The processes that follow this depend upon strategic factors. If discriminability is high enough, or the target and background well enough learned, then the subject might respond on the basis of the first stage. Otherwise, elements exceeding a certain value of "targetlikeness" can be sequentially examined. The serial examination is self-terminating and may be quite slow, compared with the actual display size slopes observed in response latencies. In general, negative responses are contingent on examination of the entire list. As Hoffman points out, work in other paradigms (e.g., Colegate, Hoffman, \& Eriksen, 1973) suggests that attention switching takes up to $200 \mathrm{msec}$, and he was able to satisfactorily fit several data sets without requiring much faster switching than this. This is a major advan- tage, given the fact that rapid sequential scanning seems a priori unlikely, considering what is known about the nervous system (Anderson, 1977).

Plainly, Hoffman's model predicts that adding similar items should delay detection in the second stage, since the similar items often receive consideration at Stage 2, prior to consideration of the target. The effect of similar distractors would be larger for negative than for positive trials, since exhaustive processing at Stage 2 would precede the negative response. Finally, in the paradigm of Experiment 2, the latency for detection of one target should be affected equally by the presence of distractors similar to either that target or the other (absent) target, fitting the data. In these respects, then, Hoffman's model receives strong support from the present data. The model is also consistent with the general trend for similar distractors to increase the rate of both misses and false alarms, although the present design was not designed to be optimal for characterizing error rate effects. Less obvious is why the function relating RTs to number of similar distractors should be negatively accelerated. One alternative is that the size of the candidate set checked at Stage 2 is actually limited to several items, even if a large number of items exceeds the preset criterion for admission. This might be a reasonable adaptation on the part of the subjects to the pressure for speed.

The present results suggest that several directions for future research might be profitable. One would be to examine performance in these tasks with displays that are curtailed by masks; this manipulation might preclude checking, and thereby allow strong tests of the checking hypothesis to be carried out. The masking could be manipulated on a mixed-trial basis, so that strategic adjustments would not be possible. An additional question of interest concerns the relationship between the discriminability variable employed here, and standard manipulations of "visual quality" (e.g., contrast reduction or superimposing of visual noise on a display). Visual quality effects are additive with the effects of display size in search; this turns out to be a very robust result (Logan, 1978; Pashler \& Badgio, 1985). The striking difference between the effects of target-distractor discriminability and visual quality changes in search seems like it might be a useful clue to the organization of the underlying system, but its implications remain to be elucidated.

\section{REFERENCES}

ANDERSON, J. A. (1977). Neural models with cognitive implications. In D. LaBerge \& S. Samuels (Eds.), Basic processes in reading: Perception and comprehension (pp. 27-90). Hillsdale, NJ: Eribaum.

Colegate, R. L., Hoffman, J. E., Eriksen, C. W. (1973). Selective encoding from multielement visual displays. Perception \& Psychophysics, 14, 217-224.

Corcoran, D. W. J., Jackson, A. (1977). Basic processes and strategies in visual search. In S. Dornic (Ed.), Attention and performance VI. Hillsdale, NJ: Erlbaum.

Duncan, J. (1980a). The demonstration of capacity limitation. Cognitive Psychology, 12, 75-96. 
Duncan, J. (1980b). The locus of interference in the perception of simultaneous stimuli. Psychological Review, 87, 272-300.

DunCan, J. (1983). Category effects in visual search: A failure to replicate the "oh-zero"' phenomenon. Perception \& Psychophysics, 34, 221-232.

Egeth, H., SANTEe, J. L. (1981). Conceptual and perceptual components of interletter inhibition. Journal of Experimental Psychology: Human Perception \& Performance, 7, 506-517.

ERIKSEN, C. W., SPENCER, T. (1969). Rate of information processing in visual perception: Some results and methodological considerations. Journal of Experimental Psychology Monograph, 79 (2, Part 2).

EsTEs, W. K. (1972). Interactions of signal and background variables in visual processing. Perception \& Psychophysics, 12, 278-286.

ESTES, W. K. (1982). Similarity-related channel interactions in visual processing. Joumal of Experimental Psychology: Human Perception \& Performance, 8, 353-380.

ESTES, W., TAYLOR, H. (1964). A detection method and probabilistic models for assessing information processing from brief visual displays. Proceedings of the National Academy of Science, 52, 446-454.

GARDNER, G. T. (1972). Evidence for independent parallel channels in tachistoscopic perception. Cognitive Psychology, 4, 130-155.

GiLmore, G. C. (1980). Letter interactions in brief visual displays. Quarterly Joumal of Experimental Psychology, 32, 649-668.

HoFman, J. E. (1978). Search through a sequentially presented visual display. Perception \& Psychophysics, 23, 1-11.

Hofrman, J. E. (1979). A two-stage model of visual search. Perception \& Psychophysics, 25, 319-327.

KINCHLA, R. A. (1974). Detecting target elements in multi-element arrays: A confusability model. Perception \& Psychophysics, 15, 149-158.

KRUEGER, L. (1984). Self-termination in same-different judgements:
Multiletter comparison with simultaneous and sequential presentation. Joumal of Experimental Psychology: Learning, Memory, \& Cognition, 10, 271-285.

LOGAN, G. (1978). Attention in character classification tasks: Evidence for the automaticity of component stages. Journal of Experimental Psychology: General, 107, 32-63.

LoGAN, G. D., ZBrodofF, N. J. (1982). Constraints on strategy construction in a speeded discrimination task. Journal of Experimental Psychology: Human Perception \& Performance, 8, 502-520.

Logan, G. D., Zbrodoff, N. J., Fostey, A. (1983). Costs and benefits of strategy construction in a speeded discrimination task. Memory \& Cognition, 11, $485-493$.

PASHler, H., \& BADGio, P. (1985). Visual attention and stimulus identification. Journal of Experimental Psychology: Human Perception \& Performance, 11, 105-121.

Santee, J. L., \& EgeTH, H. E. (1982). Do reaction time and accuracy measure the same aspects of letter recognition? Joumal of Experimental Psychology: Human Perception \& Performance, 8, 489-501.

ShIFfrin, R. M., \& GARDNER, G. T. (1972). Visual processing capacity and attentional control. Journal of Experimental Psychology, 93, $72-83$.

ShIFFrin, R. M., \& GeIsLeR, W. S. (1973). Visual recognition in a theory of information processing. In R. L. Solso (Ed.), The Loyola Symposium: Contemporary viewpoints in cognitive psychology. Washington, DC: Winston.

van der Heuden, A. H. C., Malhas, M. S. M., \&an den Roovart, B. P. (1984). An empirical interletter confusion matrix for continuous line capitals. Perception \& Psychophysics, 35, 85-88.

(Manuscript received August 1, 1986;

revision accepted for publication December $19,1986$. 